\title{
Malignant mixed Müllerian tumour of the uterus associated with non-islet cell tumour hypoglycaemia
}

José Ignacio Martínez-Montoro ${ }^{1}$, Beatriz Fernández-Medina ${ }^{1}$, Pedro Medina-Delgado ${ }^{2}$, Francisco J. Tinahones ${ }^{1,3,4}$

1Department of Endocrinology and Nutrition, Virgen de la Victoria University Hospital, Málaga, Spain

2Department of Internal Medicine, Virgen de la Victoria University Hospital, Málaga, Spain

${ }^{3}$ Laboratorio de Investigación, Instituto de Investigación Biomédica de Málaga (IBIMA), Spain

${ }^{4}$ Centro de Investigación Biomédica en Red de la Fisiopatología de la Obesidad y Nutrición (CIBEROBN), Instituto de Salud Carlos III (ISCIII), Madrid, Spain

Submitted: 27 March 2021, accepted: 18 July 2021

Online publication: 30 July 2021

Arch Med Sci 2021; 17 (5): 1433-1435

DOI: https://doi.org/10.5114/aoms/140296

Copyright $\odot 2021$ Termedia \& Banach

Non-islet cell tumour hypoglycaemia (NICTH) is a very uncommon cause of hypoglycaemia due to increased secretion of insulin-like growth factor-2 (IGF-2) and its precursors as a result of overexpression of the IGF2 gene in the tumour, which can activate the insulin receptor [1, 2]. Malignant mixed Müllerian tumour of the uterus (MMMTU) constitutes a particularly rare and aggressive entity that typically presents with the classical triad of symptoms consisting of pain, severe vaginal bleeding and the passage of necrotic tissue per vaginum [3]. We report for the first time a case of MMMTU including hypoglycaemia in the setting of NICTH as a presenting manifestation.

A 72-year-old woman presented with abdominal pain and distension, weight loss, mass in the abdomen and occasional vaginal bleeding for 1 month. She also reported sporadic episodes of dizziness, blurred vision and sweating that remitted after the intake of carbohydrates. The patient had a history of rectal adenocarcinoma that was surgically removed (low anterior resection) and received adjuvant chemoradiotherapy 11 years ago. There was no history of diabetes mellitus, alcohol abuse or illicit drug use; the only medication the patient was taking was omeprazole $20 \mathrm{mg}$ daily and paracetamol, which were prescribed after the presentation of these symptoms. Physical examination revealed a palpable mass on the infraumbilical midline. Chest- $X$ ray showed multiple, bilateral pulmonary nodules compatible with metastatic disease; abdominal ultrasound identified a $16.5 \times 10 \times 17 \mathrm{~cm}$ heterogeneous pelvic mass with internal vascularity and grade II/IV ureteral dilatation due to the extrinsic compression of the mass. In the emergency room, the patient had an episode of intense weakness, sweating and tremors with capillary blood glucose of $40 \mathrm{mg} / \mathrm{dl}$ that relieved after the administration of intravenous 50\% dextrose. In the routine biochemistry we found elevated circulating levels of CA-125 (80 U/ml), creatine kinase (488 U/l), lactate dehydrogenase $(717 \mathrm{U} / \mathrm{l})$ and C-reactive protein $(142.3 \mathrm{mg} / \mathrm{l})$, as well as low blood glucose levels ( $47 \mathrm{mg} / \mathrm{dl})$. Transaminase levels were normal, as were bilirubin and $\gamma$-glutamyl transferase. There were no alterations in the haemogram, coagulation or the urine test.

\author{
Corresponding authors: \\ José Ignacio Martínez-Montoro \\ Francisco J. Tinahones \\ Department \\ of Endocrinology \\ and Nutrition \\ Virgen de la Victoria \\ University Hospital \\ 29010 Málaga, Spain \\ E-mail: \\ joseimartinezmontoro@ \\ gmail.com, \\ fjtinahones@uma.es
}


Table I. Laboratory testing for assessment of hypoglycaemia

\begin{tabular}{|lc|}
\hline Parameter & $\begin{array}{c}\text { Measured } \\
\text { value }\end{array}$ \\
\hline Plasma glucose $(70-100 \mathrm{mg} / \mathrm{dl})$ & 37 \\
\hline Insulin $(3-25 \mu \mathrm{U} / \mathrm{ml})$ & $<0.1$ \\
\hline Proinsulin $(3-20 \mathrm{pmol} / \mathrm{l})$ & 0.7 \\
\hline C-peptide $(0.81-3.85 \mathrm{ng} / \mathrm{ml})$ & 0.09 \\
\hline -hydroxybutyrate $(<3.1 \mathrm{mg} / \mathrm{dl})$ & $<1$ \\
\hline Insulin antibodies $(<10 \mathrm{U} / \mathrm{ml})$ & Negative \\
\hline Urine oral antidiabetic drugs screen & Negative \\
\hline Serum oral antidiabetic drugs screen & Negative \\
\hline GH $(0.06-6.88 \mathrm{ng} / \mathrm{ml})$ & 0.29 \\
\hline IGF-1 $(64-188 \mathrm{ng} / \mathrm{ml})$ & 45 \\
\hline IGF-2 $(350-1000 \mathrm{ng} / \mathrm{ml})$ & 549 \\
\hline IGF-2/IGF-1 ratio $(3: 1)$ & 12.2 \\
\hline IGFBP-3 $(2.2-7.8 \mu g / \mathrm{ml})$ & 0.7 \\
\hline ACTH $(5-50 \mathrm{pg} / \mathrm{ml})$ & 24 \\
\hline Cortisol $(5-25 \mu \mathrm{g} / \mathrm{dl})$ & 47.1 \\
\hline
\end{tabular}

GH - growth hormone, IGF - insulin-like growth factor, IGBP-3 -insulin-like growth factor binding protein-3, ACTH-adrenocorticotropic hormone.

On further evaluation, abdominal and pelvic computed tomography scan located the previously described solid mass in the uterus, in close contact with the small intestine, posterior bladder wall and anterior wall of the rectum, infiltrating the mentioned organs, in addition to numerous retroperitoneal lymphadenopathies, peritoneal carcinomatosis and focal metastatic liver lesions. There was also evidence of disseminated disease in the chest computed tomography scan (multiple bilateral nodules).

During hospitalization, the patient suffered from several episodes of symptomatic hypoglycaemia, four of them with capillary blood glucose $<30 \mathrm{mg} / \mathrm{dl}$, including one with loss of consciousness. Blood glucose levels rose after the administration of $50 \%$ dextrose; however, recurrent hypoglycaemia persisted despite continuous infusion of $20 \%$ dextrose. Specific laboratory testing for the evaluation of hypoglycaemia showed low insulin, proinsulin, C-peptide and $\beta$-hydroxybutyrate; undetectable levels of insulin antibodies; negative oral antidiabetic drugs screen in both serum and urine and an IGF-2/IGF-1 ratio of 12.2 (Table I), which was concordant with the diagnosis of NICTH. Cervical biopsy was performed, with the result of a malignant mixed Müllerian tumour without evidence of a heterologous component (Figure 1). The patient declined chemotherapy and was started with prednisone $40 \mathrm{mg}$ daily with resolution of hypoglycaemia. However, she suffered progressive deterioration of her general condition and ultimately died from respiratory failure.
NICTH has been previously reported in a wide variety of benign and malignant tumours, especially among those of mesenchymal or hepatic origin. Although the most frequent histopathology corresponds to solitary fibrous tumour, mesothelioma and hepatocellular carcinoma, different tumours have been associated with this syndrome, such as adenocarcinomas, gastrointestinal stromal tumours, Burkitt's lymphoma, adrenal cortical carcinoma and medullary thyroid carcinoma [1]. We report the first case of NICTH associated with an MMMTU, a rare tumour composed of epithelial and mesenchymal elements. Our case exhibits the typical NICTH laboratory pattern: hypoglycaemia with low levels of insulin, proinsulin, C-peptide and $\beta$-hydroxybutyrate, accompanied by an IGF-2/ IGF-1 molar ratio above the normal range (3:1), usually > 10 [1]. IGF-2 levels can be normal, mainly due to elevated circulating levels of abnormally processed IGF-2 (big IGF-2) that are difficult to detect; nevertheless, IGF-1 levels are supressed, and an IGF-2/IGF-1 ratio > 10 confirms the diagnosis of NICTH $[1,4]$. Surgical removal of the tumour is considered as the definitive treatment of NICTH, though alternative options such as high-dose glucocorticoid therapy may control hypoglycaemia through a reduction in IGF-2 levels [5].

\section{Acknowledgments}

We would like to thank Juana Isabel Cid Mañas, Department of Pathological Anatomy, Virgen de la Victoria University Hospital, for her collaboration in providing the histopathological images included in the manuscript.

This study was supported by "Centros de Investigación Biomédica en Red" (CIBER) of "Instituto de Salud Carlos III" (ISCIII) (CB06/03/0018) and research grants from the ISCIII (PI18/01160), and co-financed by the European Regional Development Fund (ERFD).

\section{Conflict of interest}

The authors declare no conflict of interest. 

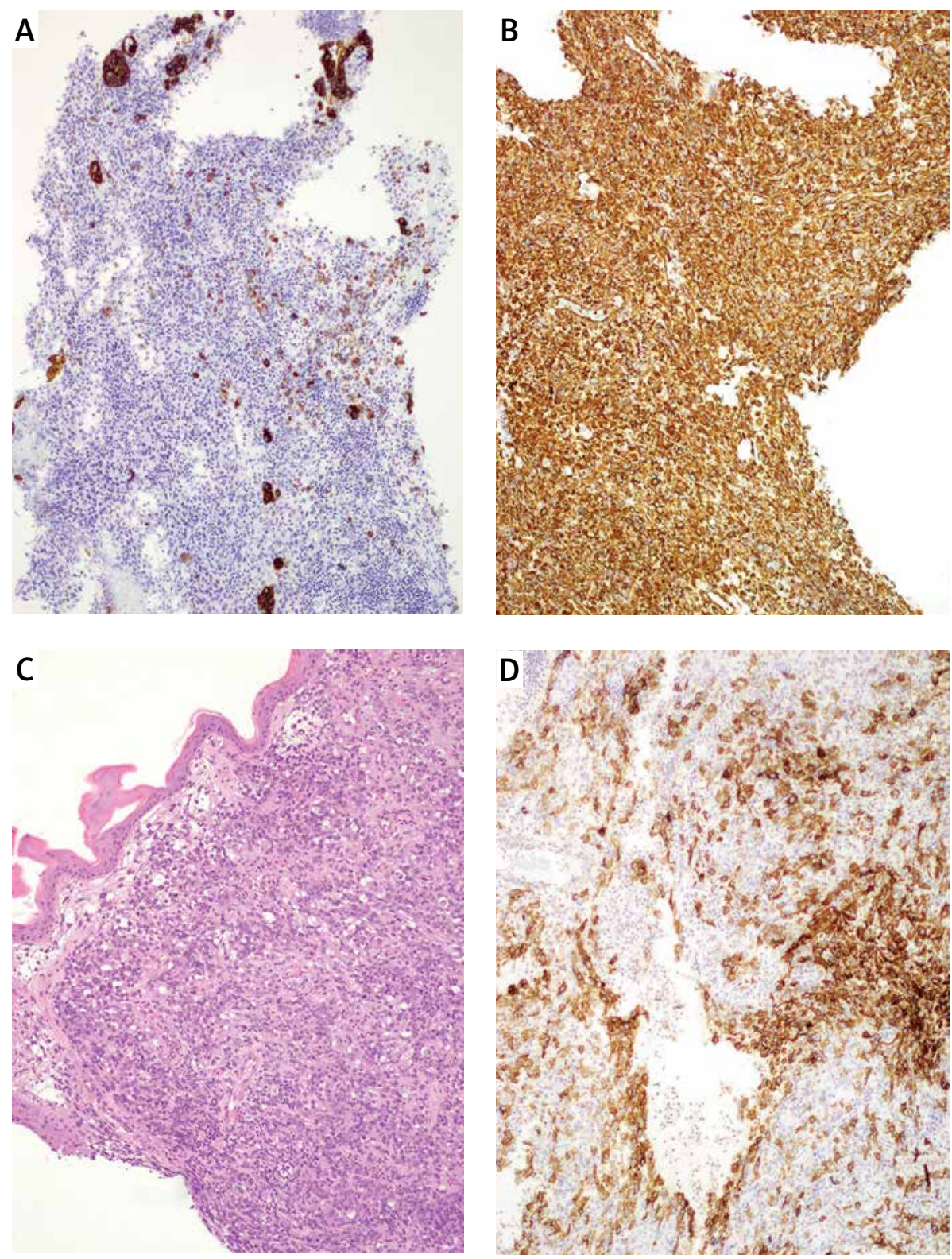

Figure 1. Uterine cervix biopsy showing a biphasic malignant neoplasm consistent with a homologous MMMTU. A - Haematoxylin \& eosin stain of an undifferentiated malignant uterine neoplasm. B - Cytokeratin 7-positive staining of the malignant epithelial component of the tumour. C - Patchy CD10 expression of the malignant mesenchymal component of the tumour. D - Diffuse and strong vimentin-positive staining of the mesenchymal component of the neoplasm

\section{References}

1. Dynkevich Y, Rother KI, Whitford I, et al. Tumors, IGF-2, and hypoglycemia: insights from the clinic, the laboratory, and the historical archive. Endocr Rev 2013; 34: 798-826.

2. Le Roith D. Seminars in medicine of the Beth Israel Deaconess Medical Center. Insulin-like growth factors. N Engl J Med 1997; 336: 633-40.

3. Grasso S, Loizzi V, Minicucci V, et al. Malignant mixed müllerian tumour of the uterus: analysis of 44 cases. Oncology 2017; 92: 197-204.
4. Garla V, Sonani H, Palabindala V, Gomez-Sanchez C, Subauste J, Lien LF. Non-islet cell hypoglycemia: case series and review of the literature. Front Endocrinol 2019; 10: 316.

5. Bodnar TW, Acevedo MJ, Pietropaolo M. Management of non-islet-cell tumor hypoglycemia: a clinical review. J Clin Endocrinol Metab 2014; 99: 713-22. 